\title{
DIOPHANTINE APPROXIMATION PROPERTIES OF CERTAIN INFINITE SETS
}

\author{
BY \\ WOLFGANG M. SCHMIDT ${ }^{1}$
}

\begin{abstract}
We exhibit various infinite sets of reals whose finite subsets do not have good simultaneous rational approximations. In particular there is an infinite set such that each finite subset is "badly approximable" in the sense that Dirichlet's theorem is best possible up to a multiplicative constant.
\end{abstract}

1. Introduction. Recall that according to Dirichlet's theorem on linear forms, for any $n$-tuple $\left(\xi_{1}, \ldots, \xi_{n}\right)$ of reals there are infinitely many nonzero $n$-tuples $\left(q_{1}, \ldots, q_{n}\right)$ of integers with

$$
\left\|q_{1} \xi_{1}+\cdots+q_{n} \xi_{n}\right\|<q^{-n}
$$

where $q=\max \left(\left|q_{1}\right|, \ldots,\left|q_{n}\right|\right)$, and where $\|\cdots\|$ denotes the distance to the nearest integer. For different $n$-tuples of reals, this may be improved to varying degrees. Given a function $\varphi(q)$, we will say that $\left(\xi_{1}, \ldots, \xi_{n}\right)$ is $\varphi$-approximable if there are infinitely many nonzero integer $n$-tuples $\left(q_{1}, \ldots, q_{n}\right)$ with

$$
\left\|q_{1} \xi_{1}+\cdots+q_{n} \xi_{n}\right\|<q^{-n} \varphi(q) .
$$

We will call an $n$-tuple of reals a Liouville $n$-tuple if it is $\varphi$-approximable for $\varphi(q)=q^{-K}$ for every exponent $K>0$. Following the usage in [8] we will call an $n$-tuple very well approximable if it is $\varphi$-approximable with $\varphi(q)=q^{-\delta}$ and some fixed $\delta=\delta\left(\xi_{1}, \ldots, \xi_{n}\right)>0$. On the other hand an $n$-tuple is called badly approximable if it is not $\varphi$-approximable for any $\varphi(q)$ which tends to zero.

An $n$-tuple is not $\varphi$-approximable if

$$
\left\|q_{1} \xi_{1}+\cdots+q_{n} \xi_{n}\right\| \geqslant q^{-n} \varphi(q)
$$

with at most finitely many exceptions $\left(q_{1}, \ldots, q_{n}\right)$. It is not Liouville precisely if there is an $H=H\left(\xi_{1}, \ldots, \xi_{n}\right)$ such that

$$
\left\|q_{1} \xi_{1}+\cdots+q_{n} \xi_{n}\right\|>q^{-H}
$$

with at most finitely many exceptions. An $n$-tuple is not very well approximable precisely if for each $\delta>0$,

$$
\left\|q_{1} \xi_{1}+\cdots+q_{n} \xi_{n}\right\|>q^{-n-\delta}
$$

Received by the editors June 29, 1982.

1980 Mathematics Subject Classification. Primary 10F10; Secondary 10K15.

'Supported by NSF grant NSF-MCS-8015356. 
with at most finitely many exceptions depending on $\delta$. By the transference principle (Khintchine [4]; expositions in Cassels [2] and Schmidt [8]), this holds if and only if

$$
\max \left(\left\|q \xi_{1}\right\|, \ldots,\left\|q \xi_{n}\right\|\right)>q^{-(1 / n)-\varepsilon}
$$

for each $\varepsilon>0$ and $q>q_{0}(\varepsilon)$. An $n$-tuple is badly approximable precisely if there is a $c=c\left(\xi_{1}, \ldots, \xi_{n}\right)>0$ such that

$$
\left\|q_{1} \xi_{1}+\cdots+q_{n} \xi_{n}\right\|>c q^{-n} .
$$

By the transference principle this is equivalent to the existence of a $c^{\prime}=c^{\prime}\left(\xi_{1}, \ldots, \xi_{n}\right)$ $>0$ such that for $q>0$ we have

$$
\max \left(\left\|q \xi_{1}\right\|, \ldots,\left\|q \xi_{n}\right\|\right)>c^{\prime} q^{-1 / n} .
$$

THEOREM 1. For $0<\lambda \leqslant 1$ put

$$
\xi(\lambda)=\sum_{t=1}^{\infty} 2^{-\left[2^{t+\lambda}\right]},
$$

where $[\ldots]$ denotes the integer part. Then any finite $\operatorname{set}\left(\xi\left(\lambda_{1}\right), \ldots, \xi\left(\lambda_{n}\right)\right)$ with distinct $\lambda_{1}, \ldots, \lambda_{n}$ is not a Liouville $n$-tuple.

In particular there is a set $\mathfrak{X}_{1}$ of reals of the cardinality of the continuum such that no (nonempty) finite subset is Liouville. This answers a question ${ }^{2}$ of L. Asam which got me started on this work. Now Liouville $n$-tuples have Hausdorff dimension only $n-1$ and therefore are quite rare, which makes the existence of $\mathfrak{X}_{1}$ relatively likely. The very well approximable $n$-tuples are not quite as rare since their Hausdorff dimension is $n$, but they still are fairly rare since their Lebesgue measure is 0 .

THEOREM 2. Suppose that for each natural $n$ we are given a positive function $\varphi_{n}$ with

$$
\sum_{q=1}^{\infty} q^{-1} \varphi_{n}(q)<\infty .
$$

Then there exists a set $\mathfrak{X}_{2}=\mathfrak{X}_{2}\left(\varphi_{1}, \varphi_{2}, \ldots\right)$ of reals of the cardinality of the continuum, such that for any $n$, every $n$-tuple of distinct elements of $\mathfrak{X}_{2}$ is not $\varphi_{n}$-approximable.

In particular, there exists a set $\mathfrak{X}_{2}^{*}$ of the cardinality of the continuum such that no finite subset is very well approximable.

I am unable to exhibit a set $\mathfrak{X}_{2}^{*}$ with this property which is as explicit as the set $\mathfrak{X}_{1}$. The proof of Theorem 2 will involve an easy application of a theorem of Mycielski about the Lebesgue measure.

The situation becomes more difficult without hypothesis (1.1). Our next theorem supersedes Theorem 2, which was stated separately only because its proof is shorter.

THEOREM 3. Suppose we are given functions $\varphi_{1}, \varphi_{2}, \ldots$ with

$$
\lim _{q \rightarrow \infty} \varphi_{n}(q)=0 \quad(n=1,2, \ldots) .
$$

Then there exists a set $\mathfrak{X}_{3}=\mathfrak{X}_{3}\left(\varphi_{1}, \varphi_{2}, \ldots\right)$ of reals of the cardinality of the continuum such that for any $n$, each subset of $n$ elements is not $\varphi_{n}$-approximable.

\footnotetext{
${ }^{2}$ Letter dated June 22, 1981.
} 
Under hypothesis (1.1), the $\varphi_{n}$-approximable $n$-tuples have measure zero. But when (1.1) does not hold, then almost every $n$-tuple is $\varphi_{n}$-approximable (Khintchine [5]; for an exposition see Cassels [2] or Schmidt [8]), which makes the "construction" of $\mathfrak{X}_{3}$ more difficult. In view of the transference principle, Theorem 3 is equivalent to

THEOREM 3'. Given $\varphi_{1}, \varphi_{2}, \ldots$ as in Theorem 3 , there is a set $\mathfrak{X}_{3}^{\prime}$ of the cardinality of the continuum such that for each $n$ and for distinct elements $\xi_{1}, \ldots, \xi_{n}$ of $\mathfrak{X}_{3}^{\prime}$ we have

$$
\max \left(\left\|q \xi_{1}\right\|, \ldots,\left\|q \xi_{n}\right\|\right)>q^{-1 / n} \varphi_{n}(q)
$$

for $q>q_{0}\left(\xi_{1}, \ldots, \xi_{n}\right)$.

We are unable to decide whether there exists a set $\mathfrak{X}_{4}$ of the cardinality of the continuum such that every finite subset is badly approximable. But we do have

THEOREM 4. There exists an infinite set $\mathfrak{X}_{4}$ of reals such that every finite subset is badly approximable.

The proof of Theorem 4 will use the $(\alpha, \beta)$-games introduced in [7] and will be fairly simple. The proof of the more difficult Theorem 3 will also involve games and will be given at the end.

2. Proof of Theorem 1. Suppose that $0<\lambda_{1}<\cdots<\lambda_{n} \leqslant 1$, and put $\xi_{i}=\xi\left(\lambda_{i}\right)$ $(i=1, \ldots, n)$. For natural $s$,

$$
\left\|2^{2^{s}} \xi_{i}\right\|=\left\|2^{2^{s}} \sum_{t=s}^{\infty} 2^{-\left[2^{t+\lambda_{i}}\right]}\right\| \quad(i=1, \ldots, n) .
$$

Now

$$
2^{s+\lambda_{i}}-2^{s}=\left(2^{\lambda_{i}}-1\right) 2^{s}=\mu_{i} 2^{s},
$$

say, with $0<\mu_{1}<\cdots<\mu_{n} \leqslant 1$, and therefore

$$
\left\|2^{2^{s}} \xi_{i}\right\| \gg \ll 2^{-\mu_{i} 2^{s}} \quad(i=1, \ldots, n)
$$

for large $s$. Here $\ll$ is the well-known Vinogradov symbol, and $f(s) \gg \ll g(s)$ signifies that both $f(s) \ll g(s)$ and $g(s) \ll f(s)$.

Suppose that $\left(q_{1}, \ldots, q_{n}\right)$ with large maximum modulus $q$ is given. By using induction on $n$ in the proof of our theorem, we may suppose that $q_{1} \neq 0$. Choose $s$ with

$$
2^{\rho 2^{s-1}} \leqslant q<2^{\rho 2^{s}}
$$

where $\rho=\min \left(\frac{1}{2} \mu_{1}, \frac{1}{2}\left(\mu_{2}-\mu_{1}\right)\right)$ when $n>1$, but $\rho=\frac{1}{2} \mu_{1}$ when $n=1$. As $q$ tends to infinity, then so does $s$, and

$$
\left|q_{1}\right| 2^{-\mu_{1} 2^{s}} \leqslant q 2^{-\mu_{1} 2^{s}}=o(1) .
$$

It follows that

$$
\left\|2^{2^{s}} q_{1} \xi_{1}\right\| \gg \ll\left|q_{1}\right| 2^{-\mu_{1} 2^{s}} \gg 2^{-\mu_{1} 2^{s}}
$$

On the other hand, when $n>1$, then also

$$
\left\|2^{2^{s}} q_{i} \xi_{i}\right\| \ll q 2^{-\mu_{i} 2^{s}} \ll q 2^{-\mu_{2} 2^{s}}=o\left(2^{-\mu_{1} 2^{s}}\right) \quad(i=2, \ldots, n)
$$


by (2.1) and by the choice of $\rho$. As a consequence,

$$
\left\|2^{2^{s}}\left(q_{1} \xi_{1}+\cdots+q_{n} \xi_{n}\right)\right\| \gg 2^{-\mu_{1} 2^{s}}
$$

and hence

$$
\left\|q_{1} \xi_{1}+\cdots+q_{n} \xi_{n}\right\| \gg 2^{-\left(1+\mu_{1}\right) 2^{s}} \geqslant 2^{-2^{s+1}} \geqslant q^{-4 / \rho} .
$$

It follows that for large values of $q$,

$$
\left\|q_{1} \xi_{1}+\cdots+q_{n} \xi_{n}\right\|>q^{-5 / \rho} .
$$

3. Proof of Theorem 2. Under hypothesis (1.1), the $\varphi_{n}$-approximable $n$-tuples are a subset of $\mathbf{R}^{n}$ of Lebesgue measure zero. Hence Theorem 2 is a consequence of the following

Proposition 1. Suppose that for each $n \geqslant 1$ we are given a subset $\mathfrak{S}_{n}$ of $\mathbf{R}^{n}$ of measure zero. Then there is a set $\mathfrak{X}$ of reals of the cardinality of the continuum such that for any $n$ and any $n$ distinct elements $\xi_{1}, \ldots, \xi_{n}$ of $\mathfrak{X}$, the $n$-tuple $\left(\xi_{1}, \ldots, \xi_{n}\right) \notin \Im_{n}$.

The author had given a proof of this proposition related to the proof (given below) of Theorem 3. But he could have saved the effort, for his colleague Mycielski pointed out to him that the proposition is in fact an immediate consequence of Mycielski's main result in [6].

4. Proof of Theorem 4. It will be convenient to recall some facts about ( $\alpha, \beta)$-games. Let $0<\alpha<1,0<\beta<1$, and let $H$ be a real Hilbert space of positive dimension. The game is played by two players, called "White" and "Black". First Black picks a closed ball ${ }^{3} B_{0}$ of arbitrary radius $\rho\left(B_{0}\right)$. Then White picks a closed ball $W_{1} \subset B_{0}$ of radius $\rho\left(W_{1}\right)=\alpha \rho\left(B_{0}\right)$. Then Black picks a closed ball $B_{1} \subset W_{1}$ of radius $\rho\left(B_{1}\right)=$ $\beta \rho\left(W_{1}\right)$. And so forth, with White decreasing the radius by the factor $\alpha$ at each move, and Black by the factor $\beta$. One obtains a nested sequence $B_{0} \supset W_{1} \supset B_{1} \supset$ $W_{2} \supset \cdots$. Given a subset $S$ of $H$, the object of White is to play such that the interscetion of the balls $B_{0}, W_{1}, B_{1}, W_{2}, \ldots$ lies in $S$. The set $S$ is called $(\alpha, \beta)$-winning if White can always win, no matter what strategy is used by Black. Further $S$ is called $\alpha$-winning where $0<\alpha<1$, if it is $(\alpha, \beta)$-winning for each $\beta$ in $0<\beta<1$.

LemMa 1. Suppose $E$ is a subspace of $H$ of positive dimension. Let $S_{E}$ be a subset of $E$ and let $S_{H}$ be the set of points of $H$ whose image under orthogonal projection on $E$ lies in $S_{E}$. If $S_{E}$ is $(\alpha, \beta)$-winning in $E$, then $S_{H}$ is $(\alpha, \beta)$-winning in $H$.

PROOF. The assertion follows from the following remarks. On the one hand, if $B_{k} \subset W_{k}$ are balls in $H$ with $\rho\left(B_{k}\right)=\beta \rho\left(W_{k}\right)$, then their projections are balls $\Pi B_{k} \subset \Pi W_{k}$ with $\rho\left(\Pi B_{k}\right)=\beta \rho\left(\Pi W_{k}\right)$. On the other hand, if $B_{k}$ is a ball in $H$ and if $W_{k+1}^{\prime}$ is a ball in $E$ with $W_{k+1}^{\prime} \subset \Pi B_{k}$ and $\rho\left(W_{k+1}^{\prime}\right)=\alpha \rho\left(\Pi B_{k}\right)$, then there exists a ball $W_{k+1}$ in $H$ with $\Pi W_{k+1}=W_{k+1}^{\prime}$, with $W_{k+1} \subset B_{k}$ and $\rho\left(W_{k+1}\right)=\alpha \rho\left(B_{k}\right)$.

It was shown in $[7,8]$ that badly approximable $n$-tuples form an $\alpha$-winning set in $\mathbf{R}^{n}$ for $0<\alpha \leqslant \frac{1}{2}$. (In fact in $\S 6$ we will prove a somewhat more precise assertion.)

\footnotetext{
${ }^{3}$ Our numbering of black balls is different from [7 or $\mathbf{8}$ ].
} 
Further, the intersection of countably many $\alpha$-winning sets is $\alpha$-winning. Now let $H$ be the real Hilbert space of sequences $\xi=\left(\xi_{1}, \xi_{2}, \ldots\right)$ with convergent $\xi_{1}^{2}+\xi_{2}^{2}+\cdots$, and with inner product $\boldsymbol{\xi} \eta=\xi_{1} \eta_{1}+\xi_{2} \eta_{2}+\cdots$. By Lemma 1, the set of $\xi \in H$ for which $\left(\xi_{1}, \ldots, \xi_{n}\right)$ is badly approximable is $\alpha$-winning in $H$. The same is true if $\xi_{1}, \ldots, \xi_{n}$ is replaced by $\xi_{i_{1}}, \ldots, \xi_{i_{n}}$ with distinct $i_{1}, \ldots, i_{n}$. Since the natural numbers have only countably many finite subsets, we obtain

Proposition 2. The set of $\xi \in H$ such that every (nonempty) finite set of components is badly approximable is $\alpha$-winning for $0<\alpha \leqslant \frac{1}{2}$.

Theorem 4 follows.

5. The game-plan for Theorem 3. We will actually prove the dual Theorem $3^{\prime}$. Throughout, $\varphi_{1}, \varphi_{2}, \ldots$ will be fixed functions with (1.2).

Given a vector $\boldsymbol{\eta}=\left(\eta_{1}, \eta_{2}, \ldots\right) \in \mathbf{R}^{\infty}$, where $\mathbf{R}^{\infty}$ consists of all real sequences, write $\boldsymbol{\eta}^{(l)}$ for the truncated vector $\boldsymbol{\eta}^{(l)}=\left(\boldsymbol{\eta}_{1}, \ldots, \boldsymbol{\eta}_{l}\right) \in \mathbf{R}^{l}$. Now if $\boldsymbol{\eta}_{1}, \ldots, \boldsymbol{\eta}_{n}$ are linearly independent, let $k\left(\boldsymbol{\eta}_{1}, \ldots, \boldsymbol{\eta}_{n}\right)$ be the least number $k$ such that $\boldsymbol{\eta}_{1}^{(k)}, \ldots, \boldsymbol{\eta}_{n}^{(k)}$ are linearly independent. But if $\boldsymbol{\eta}_{1}, \ldots, \boldsymbol{\eta}_{n}$ are linearly dependent, put $k\left(\boldsymbol{\eta}_{1}, \ldots, \boldsymbol{\eta}_{n}\right)$ $=\infty$. The symbol $\epsilon$ will be reserved for sequences $\epsilon=\left(\varepsilon_{1}, \varepsilon_{2}, \ldots\right)$ of zeros and ones.

Suppose now that we are given a sequence $\boldsymbol{\eta}$ with

$$
0<\eta_{l+1}<\frac{1}{2} \eta_{l} \quad(l=1,2, \ldots) .
$$

Put

$$
\xi(\boldsymbol{\epsilon})=\boldsymbol{\epsilon} \boldsymbol{\eta}=\varepsilon_{1} \eta_{1}+\varepsilon_{2} \eta_{2}+\cdots
$$

Proposition 3. There exists a sequence $\eta$ with (5.1) such that for $n \leqslant k$ and any $\epsilon_{1}, \ldots, \epsilon_{n}$ with $k\left(\epsilon_{1}, \ldots, \epsilon_{n}\right)=k$ we have

$$
\max \left(\left\|q \xi\left(\epsilon_{1}\right)\right\|, \ldots,\left\|q \xi\left(\epsilon_{n}\right)\right\|\right) \geqslant q^{-1 / n} \varphi_{n}(q)
$$

whenever $q>q_{0}(n, k)$.

In view of (5.1), the set $\mathfrak{N}$ of numbers $\xi(\epsilon)$ has the cardinality of the continuum. It contains a subset $\mathfrak{X}_{3}^{\prime}$ of the cardinality of the continuum such that each finite subset of $\mathfrak{X}_{3}^{\prime}$ is linearly independent over the rationals. But if $\xi\left(\epsilon_{1}\right), \ldots, \xi\left(\epsilon_{n}\right)$ are linearly independent over $\mathbf{Q}$, then $\epsilon_{1}, \ldots, \epsilon_{n}$ are linearly independent. The set $\mathfrak{X}_{3}^{\prime}$ has all the properties listed in Theorem $3^{\prime}$.

One can do a little bit more. The set $\mathfrak{N}$ is perfect, i.e. it is nonempty, closed, and it has no isolated points. A perfect set has the cardinality of the continuum. According to Mycielski [6, Theorem 2], every perfect set, such as $\mathfrak{N}$, in fact contains a perfect subset $\mathfrak{X}_{3}^{\prime}$ whose elements are linearly independent over $\mathbf{Q}$.

Our construction of $\boldsymbol{\eta}$ will be such that $\eta_{l}$ lies in the interval

$$
I_{l}: 2^{2-2 l}<\eta_{l}<2^{3-2 l} .
$$

Then (5.1) will certainly be satisfied. The construction will depend on an arbitrary parameter $\alpha$ in $0<\alpha<\frac{1}{2}$. It will involve a sequence of numbers $1=s(0)<s(1)<$ $s(2)<\cdots$, which, for reasons which will soon become apparent, might be called 
dimension splitting numbers. We will construct sets

$$
\begin{gathered}
W_{1}^{(1)} \supset W_{2}^{(1)} \supset \cdots \supset W_{s(1)}^{(1)}, \\
W_{s(1)+1}^{(2)} \supset W_{s(1)+2}^{(2)} \supset \cdots \supset W_{s(2)}^{(2)},
\end{gathered}
$$

where $W_{j}^{(l)}$ is a closed ball in $\mathbf{R}^{l}$ of radius

$$
\rho\left(W_{j}^{(l)}\right)=\alpha^{j} \quad(s(l-1)<j \leqslant s(l)) .
$$

The first ball $W_{s(l-1)+1}^{(l)}$ in the $l$ th row of (5.5) will have

$$
W_{s(l-1)+1}^{(l)} \subset W_{s(l-1)}^{(l-1)} \oplus I_{l},
$$

i.e. its elements $\left(\eta_{1}, \ldots, \eta_{l}\right)$ will have $\left(\eta_{1}, \ldots, \eta_{l-1}\right) \in W_{s(l-1)}^{(l-1)}$ and $\eta_{l} \in I_{l}$.

Denote the $j$ th element of the sequence of balls (5.5) by $W_{j}$ so that

$$
W_{j}=W_{j}^{(l)} \text { for } s(l-1)<j \leqslant s(l) .
$$

Write $\Pi_{n}$ for the projection on the first $n$ coordinates, i.e. $\Pi_{n}\left(\theta_{1}, \theta_{2}, \ldots\right)=$ $\left(\theta_{1}, \ldots, \theta_{n}\right)$. Finally define $\eta=\left(\eta_{1}, \eta_{2}, \ldots\right)$ by

$$
\begin{aligned}
\eta_{1} & =\bigcap_{j=1}^{\infty}\left(\Pi_{1} W_{j}\right), \\
\left(\eta_{1}, \eta_{2}\right) & =\bigcap_{j=s(1)+1}^{\infty}\left(\Pi_{2} W_{j}\right),
\end{aligned}
$$

etc. The plan is to construct the sequence (5.5) in such a way that $\boldsymbol{\eta}$ has the properties of Proposition 3.

Our construction will be entrusted to an infinite team of players $\mathfrak{B}_{1}, \mathfrak{B}_{2}, \ldots$ Here $\mathfrak{B}_{1}$ will choose $W_{1}, W_{3}, W_{5}, \ldots, \mathfrak{B}_{2}$ will choose $W_{2}, W_{6}, W_{10}, \ldots$, and in general $\mathfrak{W}_{n}$ will choose $W_{j}$ with $j \equiv 2^{n-1}\left(\bmod 2^{n}\right)$. Thus, more or less, $\mathfrak{B}_{n}$ will play an $\left(\alpha, \beta_{n}\right)$-game with

$$
\beta_{n}=\alpha^{2^{n}-1}
$$

but not quite, since the dimension increases as the game unfolds.

The responsibility of the player $\mathfrak{M}_{n}$ will be that for each $k, l$ with $n \leqslant k \leqslant l$ and each $\epsilon_{1}, \ldots, \epsilon_{n}$ with $k\left(\epsilon_{1}, \ldots, \epsilon_{n}\right)=k$, we have

$$
\max \left(\left\|q\left(\boldsymbol{\epsilon}_{1} \boldsymbol{\eta}^{(l)}\right)\right\|, \ldots,\left\|q\left(\boldsymbol{\epsilon}_{n} \boldsymbol{\eta}^{(l)}\right)\right\|\right) \geqslant q^{-1 / n} \varphi_{n}(q)
$$

whenever $q>q_{0}(n, k)$.

Since $\xi(\boldsymbol{\epsilon})=\lim _{l \rightarrow \infty}\left(\boldsymbol{\epsilon} \boldsymbol{\eta}^{(l)}\right)$, (5.3), and hence Proposition 3, will follow if each player can meet his responsibility. Hence we have to show that if the dimension splitting numbers are properly chosen, then each player $\mathfrak{W}_{n}(n=1,2, \ldots)$ can meet his responsibility, no matter how the other players play.

6. Lemmas on games. We now interrupt and return to the simpler situation when the game is played in a fixed space $\mathbf{R}^{l}$. But first we have to make some remarks about linear transformations. 
Write $|\xi|$ for the Euclidean norm of a point $\xi$. Write $\delta(\xi, \mathfrak{M})$ for the minimum distance from a point $\boldsymbol{\xi}$ to a linear manifold $\mathfrak{M}$, i.e. the minimum of $|\boldsymbol{\xi}-\boldsymbol{\eta}|$ over $\boldsymbol{\eta} \in \mathfrak{M}$. When $T$ is a linear transformation, write $|T|$ for the maximum of $|T \xi|$ over points $\boldsymbol{\xi}$ with norm 1 . Now let $T$ be a linear transformation from $\mathbf{R}^{l}$ onto $\mathbf{R}^{n}$ where $n>0$. We may decompose $\mathbf{R}^{l}$ as

$$
\mathbf{R}^{l}=N_{0} \oplus N_{1}
$$

where $N_{0}$ is the null space of $T$ and $N_{1}$ is its orthogonal complement. The restricted map $N_{1} \rightarrow \mathbf{R}^{n}$, call it $T_{1}$, is 1-1, and hence its inverse $T_{1}^{-1}: \mathbf{R}^{n} \rightarrow N_{1}$ is well defined. It is easily seen that

$$
|T|\left|T_{1}^{-1}\right| \geqslant 1
$$

Suppose $\mathfrak{M}$ is a linear submanifold (i.e. the translate of a subspace) in $\mathbf{R}^{n}$. Let $T^{-1} \mathfrak{M}$ be its inverse image under $T$, so that $T^{-1} \mathfrak{M}$ is a linear submanifold in $\mathbf{R}^{l}$. We claim that for each $\boldsymbol{\xi} \in \mathbf{R}^{l}$ we have

$$
\delta\left(\xi, T^{-1} \mathfrak{M}\right) \leqslant\left|T_{1}^{-1}\right| \delta(T \xi, \mathfrak{M}) .
$$

For write $\boldsymbol{\xi}=\boldsymbol{\xi}_{0}+\boldsymbol{\xi}_{1}$ according to the decomposition (6.1), and observe that $T^{-1} \mathfrak{M}=N_{0} \oplus T_{1}^{-1} \mathfrak{M}$. Then

$$
\delta\left(\xi, T^{-1} \mathfrak{M}\right)=\delta\left(\xi_{1}, T_{1}^{-1} \mathfrak{M}\right) \leqslant\left|T_{1}^{-1}\right| \delta\left(T_{1} \xi_{1}, \mathfrak{M}\right)=\left|T_{1}^{-1}\right| \delta(T \xi, \mathfrak{M}) .
$$

Now consider the $(\alpha, \beta)$-game, and suppose Black begins with a ball $B_{0}$ of radius $\rho_{0}$. Then $B_{m}$ will have radius

$$
\rho_{m}=(\alpha \beta)^{m} \rho_{0} .
$$

LEMMA 2. Let $T$ be a linear map from $\mathbf{R}^{l}$ onto $\mathbf{R}^{n}$. Suppose that $0<\alpha<\frac{1}{2}$,

$$
0<\lambda<\alpha \beta(4 n|T|)^{-1}
$$

and

$$
0<\delta<(1-2 \alpha) \lambda\left(n^{1 / 2}\left|T_{1}^{-1}\right|\right)^{-1} .
$$

Then White can play the $(\alpha, \beta)$-game in $\mathbf{R}^{l}$ in such a way that for $m=1,2, \ldots$ and each point $\left(\xi_{1}, \ldots, \xi_{n}\right) \in T W_{m}$ and each $q$ with

$$
\lambda \rho_{0}^{-1} \leqslant q^{1+(1 / n)}<\lambda \rho_{m}^{-1}
$$

we have

$$
\max \left(\left\|q \xi_{1}\right\|, \ldots,\left\|q \xi_{n}\right\|\right) \geqslant \delta q^{-1 / n} .
$$

Proof. The argument will be almost identical to that of Davenport [3], which in part goes back to Cassels [1].

We will assume that either $m=1$, or that $m>1$ and the balls $W_{1}, \ldots, W_{m-1}$ already have the desired properties. Hence White in choosing $W_{m}$ will have to worry only about numbers $q$ with

$$
\lambda \rho_{m-1}^{-1} \leqslant q^{1+(1 / n)}<\lambda \rho_{m}^{-1} .
$$


White has to play such that each $\xi \in T W_{m}$ satisfies (6.7). When (6.7) is violated, then

$$
\left|\xi_{i}-p_{i} / q\right|<\delta q^{-1-(1 / n)} \quad(i=1, \ldots, n)
$$

for a certain $n$-tuple $\left(p_{1} / q, \ldots, p_{n} / q\right)=q^{-1} \mathbf{p}$. The $n$-tuples $q^{-1} \mathbf{p}$ for which this happens with $\xi \in T B_{m-1}$, and for which $q$ lies in (6.8), will be called dangerous. We claim: The dangerous $n$-tuples are contained in a proper linear submanifold $\mathfrak{M}$ of $\mathbf{R}^{n}$.

For suppose that $q^{-1} \mathbf{p}$ is dangerous. Then (6.9) holds for some $\xi=T \boldsymbol{\eta}$ with $\boldsymbol{\eta} \in B_{m-1}$. Let $\mathbf{c}$ be the center of $B_{m-1}$ and put $\mathbf{d}=T \mathbf{c}$. Since $B_{m-1}$ has radius $\rho_{m-1}$, we have $|\mathbf{c}-\boldsymbol{\eta}| \leqslant \rho_{m-1}$ and $|\mathbf{d}-\boldsymbol{\xi}| \leqslant|T| \rho_{m-1}$. It follows that for $i=1, \ldots, n$,

$$
\begin{aligned}
\left|q d_{i}-p_{i}\right| & \leqslant\left|q \xi_{i}-p_{i}\right|+q\left|\xi_{i}-d_{i}\right|<\delta q^{-1 / n}+q|T| \rho_{m-1} \\
& \leqslant \delta\left(\rho_{m-1} / \lambda\right)^{1 /(n+1)}+|T| \rho_{m-1}\left(\rho_{m} / \lambda\right)^{-n /(n+1)} \\
& \leqslant\left(\rho_{m} / \lambda\right)^{1 /(n+1)}(\alpha \beta)^{-1}(\delta+\lambda|T|) .
\end{aligned}
$$

From (6.2), (6.4) and (6.5) we have

$$
\delta+\lambda|T|<\lambda\left|T_{1}^{-1}\right|^{-1}+\lambda|T|<\alpha \beta /(4 n)+\alpha \beta /(4 n),
$$

and therefore

$$
\left|q d_{i}-p_{i}\right|<(2 n)^{-1}\left(\rho_{m} / \lambda\right)^{1 /(n+1)} \quad(i=1, \ldots, n) .
$$

Now suppose that $\left(q^{(1)}\right)^{-1} \mathbf{p}^{(1)}, \ldots,\left(q^{(n+1)}\right)^{-1} \mathbf{p}^{(n+1)}$ are dangerous $n$-tuples. To prove our claim we have to show that the matrix $M$ with rows

$$
\left(1, p_{1}^{(j)} / q^{(j)}, \ldots, p_{n}^{(j)} / q^{(j)}\right) \quad(j=1, \ldots, n+1)
$$

has determinant zero. It will amount to the same thing to show this for the matrix $M_{1}$ with rows

$$
\left(q^{(j)}, p_{1}^{(j)}, \ldots, p_{n}^{(j)}\right) \quad(j=1, \ldots, n+1),
$$

and indeed for the matrix $M_{2}$ with rows

$$
\left(q^{(j)}, q^{(j)} d_{1}-p_{1}^{(j)}, \ldots, q^{(j)} d_{n}-p_{n}^{(j)}\right) \quad(j=1, \ldots, n+1) .
$$

Each entry in the first column of $M_{2}$ is bounded by $\left(\lambda / \rho_{m}\right)^{n /(n+1)}$, while all the other entries are bounded by (6.10). Hence

$$
\left|\operatorname{det} M_{2}\right|<(n+1) !(2 n)^{-n} \leqslant 1,
$$

and indeed det $M_{2}=0$.

Thus the dangerous points lie on a proper linear submanifold $\mathfrak{M}$ of $\mathbf{R}^{n}$. The inverse image $T^{-1} \mathfrak{M}$ is a linear submanifold of $\mathbf{R}^{l}$. White can pick $W_{m} \subset B_{m-1}$ of radius $\alpha \rho_{m-1}$ such that each point $\eta \in W_{m}$ has distance at least $(1-2 \alpha) \rho_{m-1}$ from $T^{-1} \mathfrak{M}$. Then by (6.3), the point $\xi=T \eta$ has distance at least $\left|T_{1}^{-1}\right|^{-1}(1-2 \alpha) \rho_{m-1}$ from $\mathfrak{M}$. Thus for each dangerous $q^{-1} \mathbf{p}$ we have

$$
\left|\boldsymbol{\xi}-q^{-1} \mathbf{p}\right| \geqslant\left|T_{1}^{-1}\right|^{-1}(1-2 \alpha) \rho_{m-1} \geqslant\left|T_{1}^{-1}\right|^{-1}(1-2 \alpha) \lambda q^{-1-(1 / n)},
$$

and hence

$$
\max \left(\left\|q \xi_{1}\right\|, \ldots,\left\|q \xi_{n}\right\|\right) \geqslant n^{-1 / 2}\left|T_{1}^{-1}\right|^{-1}(1-2 \alpha) \lambda q^{-1 / n}>\delta q^{-1 / n} .
$$


LEMMA 3. Consider the ( $\alpha, \beta)$-game in $\mathbf{R}^{l}$, and suppose that $0<\alpha<\frac{1}{2}$. Let $T_{1}, \ldots, T_{N}$ be linear maps from $\mathbf{R}^{l}$ onto $\mathbf{R}^{n}$. There are positive numbers $\tilde{\lambda}$ and $\tilde{\kappa}$ depending only on $\alpha, \beta, T_{1}, \ldots, T_{N}$ as follows. When

$$
\begin{aligned}
& 0<\lambda<\tilde{\lambda}, \\
& 0<\delta<\tilde{\kappa} \lambda,
\end{aligned}
$$

then White can play in such a manner that for $1 \leqslant t \leqslant N$, for $m=1,2, \ldots$ and for $\xi \in T_{t} W_{m}$, we have (6.7) for every $q$ in

$$
\lambda \rho_{0}^{-1}(\alpha \beta)^{-N} \leqslant q^{1+(1 / n)}<\lambda \rho_{0}^{-1}(\alpha \beta)^{-m} .
$$

Proof. White invites a committee (oh no!) of players $\mathfrak{W}^{(1)}, \ldots, \mathfrak{W}^{(N)}$ to make the decisions for him. Here $\mathfrak{W}^{(t)}$ chooses $W_{t}, W_{t+N}, W_{t+2 N}, \ldots$, and his duty is to ensure that (6.7) holds for $\xi \in T_{t} W_{m}$. Thus $\mathfrak{B}^{(t)}$ is playing an $\left(\alpha, \beta^{\prime}\right)$-game with $\beta^{\prime}=$ $\beta(\alpha \beta)^{N-1}$. Let $\tilde{\lambda}$ be the minimum of $\alpha \beta^{\prime}\left(4 n\left|T_{t}\right|\right)^{-1}$ and $\tilde{\kappa}$ the minimum of $(1-2 \alpha)\left(n^{1 / 2}\left|T_{t 1}^{-1}\right|\right)^{-1}$ over $t=1, \ldots, N$. By this choice of $\tilde{\lambda}, \tilde{\kappa}$ and by $(6.11)$ and (6.12), the preceding lemma applies to the game of $\mathfrak{B}^{(t)}$. Note that the $M$ th white ball in his game will be $W_{t+(M-1) N}$, and the $M$ th black ball will have radius

$$
\rho_{t, M}=\rho_{0}(\alpha \beta)^{t-1+M N} \text {. }
$$

Thus $\mathfrak{B}^{(t)}$ can play such that for $\xi \in T_{t} W_{t+(M-1) N}$, the relation (6.7) holds whenever

$$
\lambda \rho_{t, 0}^{-1} \leqslant q^{1+(1 / n)}<\lambda \rho_{t, M}^{-1} .
$$

Now when $\xi \in T_{t} W_{m}$ where $m>N$, pick $M$ with

$$
m-N+1 \leqslant t+(M-1) N \leqslant m .
$$

Then $\xi \in T_{t} W_{t+(M-1) N}$, and hence (6.7) holds for $q$ in the interval (6.15). But this interval contains the interval (6.13). The interval (6.13) is empty when $m \leqslant N$.

7. The detailed strategy. We now return to the game plan for the proof of Theorem $3^{\prime}$ as outlined in $\S 5$. The question is: What strategy should $\mathfrak{W}_{n}$ adopt for the phase of the game involving

$$
W_{s(l-1)+1}^{(l)}, \ldots, W_{s(l)}^{(l)} ?
$$

$\mathfrak{M}_{n}$ decides to "sleep" through the phases when $l=1, \ldots, n-1$. That is, he (or she) makes arbitrary moves for $W_{j}^{(l)}$ when $l<n$. But when $l \geqslant n$, he adopts a strategy indicated by Lemma 3 as follows.

We have already said that $\mathfrak{B}_{n}$ plays an $\left(\alpha, \beta_{n}\right)$-game. Given $\epsilon_{1}, \ldots, \epsilon_{n}$ with $k\left(\boldsymbol{\epsilon}_{1}, \ldots, \boldsymbol{\epsilon}_{n}\right)=k$ where $n \leqslant k \leqslant l$, the map $\boldsymbol{\eta}^{(l)} \rightarrow\left(\boldsymbol{\epsilon}_{1} \boldsymbol{\eta}^{(l)}, \ldots, \boldsymbol{\epsilon}_{n} \boldsymbol{\eta}^{(l)}\right)$ is a linear map $T$ from $\mathbf{R}^{l}$ onto $\mathbf{R}^{n}$. Corresponding to different values of $k$ in $n \leqslant k \leqslant l$ and different $\boldsymbol{\epsilon}_{1}, \ldots, \boldsymbol{\epsilon}_{n}$, we have a finite set of transformations $T_{1}, \ldots, T_{N}$ where $N=N(n, l) \leqslant 2^{n l}$. The numbers $\tilde{\lambda}=\tilde{\lambda}(n, l)$ and $\tilde{\kappa}=\tilde{\kappa}(n, l)$ can be chosen according to Lemma 3 , and according to this lemma, $\mathfrak{B}_{n}$ can deal with all the transformations $T_{1}, \ldots, T_{N}$. But what is our $\rho_{0}$, and what numbers $m$ do apply?

Let $B_{0}(l) \subset W_{s(l-1)}^{(l-1)} \oplus I_{l}$ be a closed ball in $\mathbf{R}^{l}$ of radius

$$
\rho_{0}(l)=\rho\left(B_{0}(l)\right)=\rho\left(W_{s(l-1)}^{(l-1)}\right)=\alpha^{s(l-1)} .
$$


Such a ball will certainly exist if $\rho_{0}(l) \leqslant \rho\left(I_{l}\right)=2^{1-2 l}$, hence if $s(l-1) \geqslant 2 l-1$. Now $\mathfrak{B}_{n}$ may or may not be in turn to pick the first ball in (7.1), but if he does, then he can play the game beginning with the ball $B_{0}(l)$; for then (5.6) will be satisfied. In general, $\mathfrak{W}_{n}$ will at the latest pick the $2^{n}$ th ball of the sequence (7.1), and hence the ball immediately before his turn will have radius $\rho_{0}(n, l)$ where

$$
\alpha^{s(l-1)+2^{n}}=\rho_{0}(l) \alpha^{2^{n}}<\rho_{0}(n, l) \leqslant \rho_{0}(l)=\alpha^{s(l-1)} .
$$

The number of balls in the sequence (7.1) is $s(l)-s(l-1)$, and since $\mathfrak{W}_{n}$ picks every $2^{n}$ th ball, he will pick at least $m(n, l)=2^{-n}(s(l)-s(l-1))-1$ balls. Thus when

$$
\begin{gathered}
0<\lambda_{n, l}<\tilde{\lambda}(n, l), \\
0<\delta_{n, l}<\tilde{\kappa}(n, l) \lambda_{n, l},
\end{gathered}
$$

then $\mathfrak{B}_{n}$ can ensure that for $\boldsymbol{\eta}^{(l)}$ in the intersection of (7.1), and for $\epsilon_{1}, \ldots, \epsilon_{n}$ with $k\left(\epsilon_{1}, \ldots, \epsilon_{n}\right) \leqslant l$, we have

$$
\max \left(\left\|q \boldsymbol{\epsilon}_{1} \boldsymbol{\eta}^{(l)}\right\|, \ldots,\left\|q \boldsymbol{\epsilon}_{n} \boldsymbol{\eta}^{(l)}\right\|\right)>\delta_{n, l} q^{-1 / n}
$$

whenever

$$
\lambda_{n, l} \rho_{0}(n, l)^{-1}\left(\alpha \beta_{n}\right)^{-N(n, l)} \leqslant q^{1+(1 / n)}<\lambda_{n, l} \rho_{0}(n, l)^{-1}\left(\alpha \beta_{n}\right)^{-m(n, l)} .
$$

With the notation

$$
A=1 / \alpha,
$$

this interval contains

$$
\lambda_{n, l} A^{s(l-1)+2^{n}+2^{n} N(n, l)} \leqslant q^{1+(1 / n)}<\lambda_{n, l} A^{s(l-1)+2^{n} m(n, l)},
$$

which in turn contains the interval

$$
\Im(n, l): \lambda_{n, l} A^{s(l-1)+6^{n l}} \leqslant q^{1+(1 / n)}<\lambda_{n, l} A^{s(l)-2^{n}} .
$$

We now make our final choice for $\lambda_{n, n}, \lambda_{n, n+1}, \ldots$ : these numbers should satisfy not only (7.3), but also

$$
\lambda_{n, l+1}<\lambda_{n, l} A^{-20^{n l}} \quad(l=n, n+1, \ldots) .
$$

Then the left endpoint of $\mathfrak{\Im}(n, l+1)$ will be smaller than the right endpoint of $\mathfrak{\Im}(n, l)$. We pick $\delta_{n, l}$ with (7.4). We pick the splitting numbers to increase so fast that

$$
\lambda_{n, l+1} A^{s(l+1)}>2 \lambda_{n, l} A^{s(l)} \quad(1 \leqslant n \leqslant l)
$$

for $l=0,1,2, \ldots$. Then the right endpoint of $\mathfrak{\Im}(n, l)$ will tend to infinity as $l$ goes to infinity. Thus for any $n \leqslant k$, the intervals $\mathfrak{s}(n, k), \mathfrak{\Im}(n, k+1), \ldots$ will cover a half line, say the half line $q>q_{0}(n, k)$. We further pick the splitting numbers so large that

$$
\varphi_{n}(q)<\delta_{n, l} \text { for } q \in \mathfrak{\Im}(n, l) \quad(1 \leqslant n \leqslant l) .
$$

Since the left endpoint of $\mathfrak{s}(n, l)$ can be made large by making $s(l-1)$ large, this is possible-except for the case $l=1$. But we can make (7.8) always work if retroactively we replace $\mathfrak{\Im}(1,1)$ by its right half. (This also changes $q_{0}(1,1)$.) 
Suppose now that $\boldsymbol{\eta}=\left(\eta_{1}, \eta_{2}, \ldots\right)$ is given by (5.7), (5.8), etc. For $\epsilon_{1}, \ldots, \epsilon_{n}$ and $l \geqslant k=k\left(\epsilon_{1}, \ldots, \epsilon_{n}\right)$, the estimate (7.5) holds for $q \in \mathfrak{\Im}(n, l)$. But then also (5.3) holds by (7.8). Thus (5.3) holds for $l \geqslant k$ and $q>q_{0}(n, k)$.

\section{REFERENCES}

1. J. W. S. Cassels, On a result of Marshall Hall, Mathematika 3 (1956), 109-110.

2. __ An introduction to diophantine approximation, Cambridge Tracts 45, Cambridge Univ. Press, 1957.

3. H. Davenport, $A$ note on diophantine approximation, Studies in Math. Analysis and Related Topics, Stanford Univ. Press, Stanford, Calif., 1962, pp. 77-81.

4. A. Khintchine, Über eine Klasse linearer Diophantischer Approximationen, Rend. Circ. Mat. Palermo 50 (1926), 170-195.

5. Zu Zur metrischen Theorie der diophantischen Approximationen, Math. Z. 24 (1926), 706-714.

6. J. Mycielski, Algebraic independence and measure, Fund. Math. 61 (1967), 165-169.

7. W. M. Schmidt, On badly approximable numbers and certain games, Trans. Amer. Math. Soc. 123 (1966), 178-199.

8. _ Diophantine approximation, Lecture Notes in Math., vol. 785, Springer-Verlag, Berlin and New York, 1980.

Department of Mathematics, University of Colorado, Boulder, Colorado 80309 